\title{
XXII. On the connexion of atmospheric electricity with the condensation of vapour
}

\section{William Radcliff Birt}

To cite this article: William Radcliff Birt (1850) XXII. On the connexion of atmospheric electricity with the condensation of vapour, Philosophical Magazine Series 3, 36:242, 161-171, DOI:

$10.1080 / 14786445008646455$

To link to this article: http://dx.doi.org/10.1080/14786445008646455

曲 Published online: 30 Apr 2009.

Submit your article to this journal $\sqsubset$

Џ Article views: 3

Q View related articles ๔ 
THE

LONDON, EDINBURGH AND DUBLIN

PHILOSOPHICAL MAGAZINE

A N I)

JOURNAL OF SCIENCE.

[THIRD SERIES.]

$M A R C H 1850$.

XXII. On the connexion of Atmospheric Electricity with the Condensation of Vapour. By William RADCLIfF Birt*.

TTE intimate connexion which appears to subsist between 1 the quantity of water diffused or suspended in the atmosphere, and the electric tension as exhibited by an atmospherical conductor, renders it not only interesting but important to trace either theoretically, or by means of experiment, the process of the formation of cloud and its resolution into rain, and the development of electricity as connected with each of these processes.

It is not my intention in the present paper to detail any new experiments, nor to bring before the reader the results of any recent investigations on this interesting subject, further than as they may incidentally throw light on any remarks which it may be found necessary to indulge in, in prosecuting the object now proposed. I shall consequently place before the reader, so far as my knowledge extends, what has been already written on the subject; and 1 must therefore claim his indulgence, especially in a work like this, for treading beaten ground, and presenting nothing novel to his notice. The office I propose to myself is that of a lens, which, concentrating the scattered rays of light, renders them more powerfully efficient in illuminating in some cases obscure objects.

The first writer I shall quote is John Read of Knightsbridge. In his work on atmospheric electricity published in 1793, at page 22 he has this remark relative to the relation of air to electricity: "I have no idea, much less suppose, that any, even the least quantity of electricity does ever come from air itself; for there is reason to think, that air as air, independent of the other substances, is unable to furnish any particle of

* Communicated by the Author.

Phil. Mag. S. 3. Vol. 36. No. 242. March 1850. M 


\section{$162 \mathrm{Mr}$. W. R. Birt on the connexion of Atmospheric Electricity}

this fluid; and I conjecture that pure air and electricity are actually incapable of uniting; and therefore it is that air is never known to attract, absorb, nor conduct electricity, but in proportion to the conducting substances with which it happens to be occasionally mixed : nor is air excitable of electricity by any means that is yet known; therefore air does not appear to be either an electric or a conducting body.

"It is in consequence of these peculiar properties of air that it is found to insulate the electric fluid more perfectly than any other substance."

In the above extract we are informed of the perfect indifference of air to electricity, whether we regard the latter as a fluid, or adopt the more modern view of its being a force brought into play by heat, friction, \&c. Keeping steadily in mind this perfect indifference, the following extract presents us with a mode by which the electricity, or regarding it at present as a force, "the electric force," is conveyed from one portion of the atmosphere to another:-

"The electricity rises invisibly from the earth concealed in the aqueous vapour; and as the vapour ascends higher and higher into cooler air, it becomes more condensed, by which the electricity which it contains will become more condensed also*, insomuch that it will now display its energy; and in whatever direction it moves, it is sure to move actively, animated with a penetrating and expansive force; for in this state proper instruments will easily accumulate and detain it, so as to render it visible."

In this paragraph Read says " the electricity rises invisibly from the earth concealed in the aqueous vapour;" in other words, each particle of vapour as it rises from the earth is electrified (from what source we do not now stop to inquire), or is capable, when placed in a proper situation, of exhibiting that force which we are in the habit of terming "electric." It may be well here to contemplate each particle of vapour as surrounded by pure air, through which, as air, the electric force will not pass, $i$. e. the force cannot be communicated through it unless it is of sufficient tension to "strike" through the space to another conducting body. Now if by any means heat is abstracted from the vapour particles, or they pass into a stratum of cold air, they become condensed, $i$. e. they are reduced in size, contemplating each individual drop, or several run together and produce visible vapour; in both cases the

* It is well known that if equal quantities of electricity be imparted to two unequal insulated conductors, the electricity on the least surface will become more intense than on the largest. Electrified vapour is also subject to this law. [J. Read.] 
surface over which the electric force was originally spread is reduced, so that it is by condensation confined to a smaller surface, and consequently ready to exert itself with greater energy, in other words, its tension is increased. If under these circumstances a substance capable of receiving and conducting the force is brought into contact with the vapour, the latter parts with its charge, or so much of it as is necessary to maintain an equilibrium between it and such bodies as we have just alluded to.

We have here presented to our notice two important par. ticulars connected with the ascent of electrified vapour in the process of evaporation-its insulation by the surrounding air, and the increase of electric tension upon its condensation. In connexion with the insulation of the electrified vapour, or as Howard more correctly terms it, "suspended roater (not vapour, for that is a gas and therefore a non-conductor )," Howard, in his Climate of London, vol. i. p. 148, has this important passage :-

"We can scarcely imagine a body more perfectly INSULATED than the first particle of water, which separating from vapour that has ascended into the higher atmosphere, begins to obey the law of gravity. There are two sources from whence such a particle may obtain an electric charge, viz. the surrounding $A I B$, and the vapour out of which it was formed; and which MAY (though in itself non-electric) afford to the water, now reduced many hundred fold in volume, A REAL POSITIVE CHARGE."

[The words printed in small capitals were scored by $\mathbf{M r}$. Howard, those in italics by myself.-W. K. B.]

It may be remarked in passing, that the views of these authors as to the source of the electric force differ. Read regards the force as rising from the earth. Howard conceives the vapour or aqueous gas to be destitute of electric force, but to exhibit it, or rather to receive a charge on its condensation and resumption of the form of water.

We have in the foregoing extract brought clearly before the mind a single drop of water positively electrified and perfectly insulated. Keeping this insulated electrified drop of water in view, Mr. Howard points out to us the manner in which its electric tension may be, and most probably is, increased. In his Introduction, page lxiv, in speaking of the formation of the cumulus, he says, "On these considerations we are obliged to admit as a cooperating cause of the increase of this cloud, that sort of attraction which large insulated conducting masses exercise wohen charged, on the smaller ones which lie within their infuence," viz. "to throw the small one into an 
opposite state and then attract it." This passage strikingly illustrates the increase of the electric tension by the increase of the size of the drop, not simply by the attraction of aggregation alone, but by the larger drops, considered as insulated conductors, throwing the smaller drops into an opposite state and then attracting them; so that in addition to the attraction of aggregation, by which the drops have a tendency to coalesce, the electrical attraction alluded to by Howard has a tendency to increase the size of the larger drops, or rather to produce " an agglomeration of many minute and feebly electrified globules into one rain-drop." "Thus," as Howard says in the passage which we have already quoted, "the drops of which a cumulus consists may become larger the longer it is suspended, and the electricity stronger from the comparative diminution of surface." It must, however, be understood, that the larger drops thus formed are not rain-drops in the proper acceptation of the term, the cumulus never affording rain unless a disturbance of its electrical state takes place.

Under the circumstances above referred to, the cumulus and its'particles of suspended water are regarded as charged conductors bearing, not trunsmitting, the electric force from one portion of the atmosphere to another. In this as well as the other processes that have been referred to, rain does not enter as a product. The remarks, however, of $\mathrm{Mr}$. Howard on the first formation of rain are so extremely apposite, that in tracing the electrical action which we are capable of recognizing in the atmosphere, I shall freely avail myself of them; but as the paragraphs are rather long, I must content myself with a mere reference to the pages in which they occur.

In pages 149 and 150, vol. i. of the Climate of London, Mr. Howard speaks of the first formation of rain in connexion with a double mode of the formation of cloud; viz. the condensation of vapour by refrigeration in the higher regions of the atmosphere, and the production of cumuloid masses by the condensation in the lower, of the vapour immediately evaporated from the surface of the earth. In connexion with the first mode, and in accordance with the former extracts, each particle of cloud, each minute drop of water thus separated is positively charged; and by each (thus positively charged) obeying the law of gravitation, the whole subside to a region in which they remain suspended, forming in that region a visible haze. Now I presume, from what Mr. Howard has said on the formation of cirrus, and his comparison of it to a transmitting or imperfect conductor, carrying the electric force from one portion of the atmosphere to another at a great distance over the intervening surface of the earth (Introduction, page 
lxvii and lxviii), that the resolution of this haze into the two diverse modifications (taking these, of course, as types of two distinct classes of clouds), cirrus and cirrostratus, depends entirely on the electric state of the circumambient air. If the equilibrium between the two masses of atmosphere, either superposed or on the same level and at a great distance from each other, is disturbed, the particles of water separated by condensation immediately partake (as suggested) of the nature of conductors, and are then drawn out into fine lines or pencils of condensed vapour, which transmit the electric force from one portion of the atmosphere to the other, until in some cases the equilibrium is restored. Thus cirrus is formed. On the other hand, if there is not such a disturbance of the electric state of the atmosphere as here contemplated, but the charged particles of water gradually subside so as ultimately to form, from the accumulated haze, a thin extended sheet of cloud, seen through which the heavenly bodies appear with increasing dimness, the larger luminaries being not unfiequently surrounded by well-defined halos, then the cirrostratus is formed. In the former instance, the production of cirrus, while the electric force is in process of transmission from one portion of atmosphere to another, rain is not produced. When the equilibrium is restored, and the fine lines of cirri cease to transmit the electric force, the small orbicular masses of cirrocumulus are produced; and it may be that not only each particle may retain its charge and sustain the character of an insulated conductor, but the congeries of particles of water forming the orbicular mass itself may possess such a character; the mass, as in the case of the cumulus, being an insulated conductor, possessing such an amount of electric tension as is necessary to preserve its independence among the other orbicular masses forming the entire cloud; each orbicular mass being considered as a component part thereof, just as each particle of water is a component part of a cumulus. According as these congeries of clouds may exist in dry or moist air, so evaporation or condensation may take place on their surfaces. In dry air they would gradually disappear; in moist air they would not only augment in size, but their electric tension might be so modified as to produce electric attraction, by which they might pass into the modification of cirrostratus.

In the foregoing remarks we have contemplated the passage of the condensed vapour in the higher and middle regions of the atmosphere, through the successive stages of cirrus and cirrocumulus to cirrostratus. Still keeping in mind the essential character of cirrus, and viewing its progress roithout a restoration of the equilibrium, we may inquire into the result 


\section{$166 \mathrm{Mr}$.W.R. Birt on the connexion of Atmospheric Electricity}

produced under such circumstances. In either case, whether it be a direct formation of cirrostratus from haze, or a secondary formation of this cloud from cirrus, the more perfect insulation of the charged aqueous particles which obtains in the cases of cumulus and cirrocumulus is not present. In fact the cirrostratus is a cloud more or less prevalent in a moist state of the atmosphere; and in contrasting its formation from cirrus with the formation of cirrocumulus from cirrus, it would appear that in a drier atmosphere, when cirrus has ceased to transmit the electric force, cirrocumulus results; while in a moister atmosphere the cirrus gradually subsides into cirrostratus. This origin of cirrostratus may be traced long after the clond is decidedly formed, by the striated appearance which it presents to the eye when the moon is seen behind it. Mr. Howard remarks, that "when the cirrostratus is prevalent, the lower atmosphere is usually pretty much charged with dew or haze, and therefore in a state to conduct an electric charge to the earth."

From what has preceded, we are enabled to gather, that in the case which Mr. Howard has suggested in pages 149 and 150 , the general state of the atmosphere was sufficiently moist to favour the production of cirrostratus from the haze resulting from the subsidence of the particles of water in the higher regions of the atmosphere. We may now pass on to the electrical effects consequent on the production of this sheet of haze, or its resulting sheet of cirrostratus. Mr. Howard regards the effect as the production of a negative charge in a lower stratum of the atmosphere; he says, "As soon as a sufficiently dense stratum of these particles is formed, we have the SUPERINDUCING CAUSE at the region $m, m, m$, by which the lorver air may be rendered NEGATIVE; and the accumulation of such a haze before rain is not a matter of supposition only, but of long observation." In immediate connexion with this remark of Howard, it may not be inappropriate to mention, that during my late discussion of the electrical observations at $\mathrm{Kew}$, I found that in most of the instances in which negaiive electricity was observed at Kew, cirrostratus was observed at Greenwich.

We now turn to the consideration of the production of the cumuloid masses by the condensation in the lower atmosphere of the aqueous vapour emitted by the heated earth. In connexion with this, Mr. Howard appears to regard three strata of the atmosphere as contemporaneously existing:-1st. The region of cirrostratus of course containing much aqueous vapour in a condensed state, this region possessing (or rather the cloud suspended in it) a positive charge; 2 nd, the region 
inmediately below it electrified negatively, the depth of this stratum depending upon the thickness or density of the bed of haze above; and 3rd, the lowest stratum possessing the usual positive charge of the atmosphere. It is in this lower stratum that $\mathrm{Mr}$. Howard considers the cumuloid masses to be formed; and while the negative stratum remains suspended between the superior and inferior positively electrified strata, the only result is a simple union of the cumulus below with the cirrostratus above, forming the compound modification cumulostratus. During this process no rain falls; the reason Mr. Howard appear's to regard as the absence of a transmission of the electric force; for he says, "The effect of the superinducing charge on such cloud may produce either the cUMUlostratus, which appears to be a SIMPLE UNION of clouds, or the NIMBUS, which is A UNION WITH TRANSMISSION of electricity." And it further appears from Mr. Howard's remarks, that while a stratum positively charged is interposed between the earth and the negative stratum, rain is not produced; but the moment the negative stratum reaches the earth, and the cumuloid masses are enveloped and lose their positive charge in it, "they then attract and are attracted by the positive haze (query cirrostratus) above, and the first drops of rain are [thus] formed," the cloud produced being a nimbus. "This rain opens an immediate communication with the earth; the positive electricity, which before rendered the particles buoyant, STREAMS DOWN ALONG WJTH THE RAIN AND THROUGH IT; and the shower is propagated in all directions till the whole mass of cloud is brought into action." It is worthy of remark, that generally before a shower an atmospherical conductor indicates the presence of negative electricity.

Mr. Howard appears to view the mode in which rain conducts eleciricity to the earth under two aspects:-1st. "The individual drops may receive an intense charge at the moment of their formation and during their fall through the cloud, which charge they bring to the ground." Throughout the whole of Mr. Howard's reasoning, the great increase of tension consequent on the increase in the size of the drops from the agglomeration of the minute particles of water separated by condensation is very prominent. Under ordinary circumstances the intense charge of each drop is brought to the ground; but if the quantity of electricity is increased in a greater proportion than the surface of each drop over which it is spread, then in the very act of descending to the earth the tension may be so far increased that the electric force may separate from each drop, to seek either the surface of the cloud or of the newly-formed descending body of rain, and 
an electric discharge may take place. 2nd. Mr. Howard also regards the descending body of rain as a conducting medium: he says, "the whole aggregate of floating, uniting, and falling drops from the very summit of the cloud to the ground, may form one immense conductor." He further remarks: "On the supposition that a sudden local shower is AN ATMOSPHERICAL CONDUCTOR WITH ITS FOOT ON THE EARTH, we are able to assign a satisfactory origin and use to the spreading crown, which is frequently seen above it, and in which we may discern an arrangement, tending from every side towards the dense part where the rain is formed, in a manner not required by the simple law of gravity. These rectilinear or hairy portions are the collecting points of the conductor formed in the positive haze in consequence of the destruction of the equilibrium [of its charge], which necessarily gives rise to a flow of the electricity toroards the conductor."

Upon reviewing the successive steps in the development of cloud and rain, from the first formation of the minute water particle with its feeble electric charge, to the torrent-pouring nimbus with its violent electric discharge, we have particularly to trace the formation of this cloud in a portion of atmosphere bounded above by a sheet of cirrostratus positively electrified, which rests on a stratum of air negatively electrified, this negative state being induced by the action of the positive cirrostratus, which at this time is highly charged and ready to precipitate, the clouds below being negatively electrified by induction. The atmospheric conductors are also at this time negatively electrified, in common with other bodies on or near the earth. This (according to Howard's remarks) appears to be the moment when the nimbus is formed; the disturbance, which had been produced very gradually, now makes itself distinctly felt ; the masses of cumulus, originally positive, by the superior energy of the cirrostratus, are more or less thrown into a negative state; electrical attraction between the cirrostratus and cumulus rapidly takes place, and is accompanied by the agglomeration of the minute particles of water forming the clouds; rain immediately descends, which, if the electric tension either of the cloud or of the descending rain-drops is not too intense, becomes a conducting medium, and opens an electrical communication with the earth; but if the charge of the cloud, on the other hand, becones so high as to result in a violent detonation, either before or coincident with the formation of rain, or the rain-drops themselves are incapable of retaining the charge received by them at the time of their formation, then we have with the nimbus all the phænomena of a thunder-shower. 
Mr. Howard, in his remarks on the formation of nimbus (Introduction, page lxxi), a cloud very closely allied to the cumulostratus, expresses himself in almost the precise words of the Committee of the Royal Society*. "The cirri, also, which so frequently stretch from the superior sheet upwards and resemble erected hairs, carry so much the appearance of temporary conductors of the electricity extricated by the sudden union of its minute drops into the vastly larger ones that form the rain, that one is in a manner compelled, when viewing the phænomenon, to indulge a little in electrical speculation." The sudden agglomeration alluded to by the Committee is here unmistakeable; and it would appear from the course of reasoning developed in the preceding extracts, that the greater manifestation of the electrical attraction-noticed under the head of "formation of cumulus"-consequent upon the coalescence or union of the cumulus with the cirrostratus, which is generally crowned with cirrus, is most probably-especially when combined with the pressure, in the lower part of the cloud, of the rising; and the deposition on its upper surface of the descending vapour-the occasion of the disturbance of the electrical state of the cumulus by which the rain is produced. We have, in fact, the entire process beautifully presented to our notice, commencing with the incipient separation of the earliest drops of water by the agency of a diminished temperature from the vapour rising above the vapour plane, which drops are feebly electrified and more or less perfectly insulated; the process terminating with the sudden agglomeration of these minute and feebly electrified drops into the vastly larger ones that form rain, by which the electric tension may be, and doubtless is, so enormously increased, that the electric state of the cloud and of bodies in its immediate neighbourhood are extensively disturbed; so that if the superabundant electricity should not be carried off by the conducting or rather transmitting crown of cirri, as suggested by Howard, the exhibition of electrical phænomena, as thunder and lightning, the stroke being given either by the cloud or the ruin, generally follows. The intermediate steps appear to be governed by the "two grand predisposing causes-a falling temperature and the influx of vapour." Should these not be sufficient in their extent, either above or below, to produce a formation of cirrus or an increase of cumulus, the individuals of the latter modification gradually evaporate as evening approaches, and generally give place to a serene and tranquil night; but if both these classes of clond form rapidly, cirrostratus generally shows itself, electrical action is called more * Philosophical Magazine, vol. xxxv. p. 161, foot note. 
energetically into play, and upon the formation of cumulostratus and nimbus, the rain is produced in the manner above indicated.

Thronghout the whole of the preceding remarks there is one point presented to our notice with peculiar prominence, viz. the development of the electric force in the act, more or less, of the condensation of vapour. The entire process of nubification appears to be intimately connected with this development, and the different modifications of clouds entirely dependent on the presence or absence of a disturbed electrical state, not only when the condensation is effected, but when no previous disturbed state exists it may actually be brought. about by the condensation of vapour. It may not be toc much to say, that the electricity observed in the atmosphere is principally due to, and the product of condensed vapour. We would of course not regard the condensation of vapour (if the preceding conjectures be correct) as the only source of atmospheric electricity; but we are inclined to regard it as by far the most productive; and it now only remains to connect the foregoing conjectures and reasoning with such experiments as may be calculated to throw some light on the production of the electric force by condensation.

Mr. Reuben Phillips has lately detailed in the Philosophical Magazine some very interesting experiments on the electricity of condensation. In one experiment a jet of water was passed through a jet of steam in such a way that the electricity of the water should not affect that of the steam, the water only falling on the wire-gauze of the collector; a negative charge was obtained until the force became sufficiently strong to effect a change in the kind of electricity; in other words, the jet of water exhibited precisely the same effect as the steam alone. Mr. Phillips attributes this to the drops of water collecting negative electricity from the steam: he says, "The negative charge given to the screen was I think only produced by the drops of water collecting negative electricity from the steam, much in the same way as the wire-gauze did *."

We here see the drops of water regarded in the light of insulated (?) conductors projected into the midst of condensed vapour. Each particle of this vapour is electrified negatively; and as the particles of water either come in contact with or sufficiently near to receive the charge of the particles of vapour, the charge passes to the water-drops, and through the medium of the screen affects the electrometer. It would be very interesting to know, if with a given pressure of steam, such, for instance, as would produce the greatest negative

* Page 106 of the present volume of this Journal. 
Prof. P. Tardy on a New Equation in Flydrodynamics. 171

tension, the passage of a jet of water properly guarded so as to give no electrical indications would increase the tension; in other words, would the condensation or running together of the vapour particles cause an increase of tension in the way suggested by the Committee of Physics of the Royal Society?

Mr. Phillips's experiment, that bears more particularly on the point in question, is the mixture of steam and water in a tin pipe. When the steam was introduced alone, no electrical effects were exhibited; and when the water was introduced alone, there were also no electrical effects; but when they were mixed, a positive charge was indicated by the electrometer. The experiment was thus varied: on one occasion the steam was first introduced; and upon the jet of water being projected among the particles of steain, the mixture was electrified positively: on the other occasion the water was first introduced; and upon the jet of steam being projected into the tin pipe, the mixture was also electrified positively. These two experiments appear to me to be conclusive on the subject of the development of the electric force by condensation alone, and become a link in the chain of evidence by which nearly all the varied, beautiful, grand and terrific phænomena of the atmosphere are bound together. This one simple but efficient mode of the development of the electric force appears to be fully capable of accounting, if not for the whole, for the greater portion of the phænomena of atmospheric electricity.

Kew Observatory, Feb. 6, 1950.

XXIII. Some Observations on a Nere Equation in Hydrodynamics. By Professor P. 'TARDY of Messina*.

T HAVE lately seen an article by Professor Challis in the Number of the Philosophical Magazine for last June (Supplement), "On certain points relating to the Theory of Fluid Motion," in which, in reply to a communication of M. Bertrand to the French Academy of Sciences, he returns to his favourite argument for the truth and necessity of a new equation in hydrodynamics. At the same time he does me the honour of mentioning my name, and answering an objection which I made to his analysis for the case of the motion in space of two dimensions.

I must premise that when I wrote my memoir on the movement of fluids (Sípra alcuni Punti della Teoria del Moto de' Liquidi, Firenze 1847), I had noknowledge of Professor Challis's

* Communicated by the duthor. 